Ann. Zootech., I972, 21 (3), 347-354.

\title{
ACQUISITIONS NOUVELLES CONCERNANT LA BIOCLIMATOLOGIE DES VACHES LAITIERES
}

\author{
J. HAUPTMAN \\ Institut de Recherches pour la Production animale, \\ (Académie d'Agriculture Tchèque) \\ Uhřinèves près Prague, \\ Tchécoslovaquie \\ RÉSUMÉ
}

La méthode de calorimétrie partielle pour vaches laitières permet d'effectuer des mesures de courte durée au niveau de l'épaule, de la mamelle et de l'abdomen.

La glande mammaire émet plus de chaleur que les autres parties étudiées.

Les vaches fortes productrices de lait émettent plus de chaleur au niveau de l'épaule et de l'abdomen que les vaches faibles productrices.

Cette méthode est simple et applicable dans des installations sommaires.

\section{I. - INTRODUCTION}

La création de grandes unités de production entraîne une augmentation notable de la concentration des animaux et s'accompagne de modifications des techniques d'élevage. La réalisation d'un milieu convenable est une condition indispensable à l'obtention d'une productivité suffisante du bétail. En particulier, il est nécessaire d'assurer aux animaux le microclimat voulu. La résolution de ce problème implique la connaissance précise des données physiologiques et en premier lieu les valeurs de la production de chaleur de l'organisme pour les divers états physiologiques et dans les différentes conditions de stabulation. En effet, pour une espèce donnée, les paramètres physiologiques varient considérablement suivant la race, l'âge, le type de production et les conditions de milieu. L'adaptation climatique fait également évoluer ces paramètres. Il est donc important de mesurer la production de chaleur des animaux dans les conditions habituelles de stabulation. Or, les données bioclimatologiques dont on dispose ont été obtenues pour la plupart par des méthodes 
indirectes, soit en déterminant la consommation d'oxygène d'animaux placés individuellement dans des chambres respiratoires, soit en mesurant la production de chaleur de groupes d'animaux placés dans des locaux ventilés.

Pour étudier la production de chaleur des vaches laitières dans les conditions normales de stabulation et tenir compte des variations individuelles, nous utilisons la méthode de calorimétrie partielle à l'aide d'un système de mesure calorimétrique nouvellement élaboré. Nous avons ainsi étudié les variations de la production de chaleur en fonction de la température ambiante, de la race, de la production laitière et du type de stabulation (libre ou entravée).

\section{II. - MATÉRIEL E'T MÉTHODES}

Les études ont été réalisées avec des vaches laitières de 3 races (Pie rouge tchèque, Frisonne, Ayrshive), taries ou en lactation et placées en stabulation entravée dans des étables fermées ou en stabulation libre, en particulier dans des conditions climatiques extrêmes. Toutes les vaches laitières étaient nourries de la même manière; elles recevaient une ration de base composée d'aliments grossiers, les aliments concentrés étant distribués suivant la production laitière. Les apports alimentaires ont été calculés selon les normes en vigueur.

L'émission de chaleur n'est pas uniforme sur toute la surface corporelle en raison, en particulier, des différences d'irrigation sanguine, d'intensité de perspiration insensible et de transpiration des différentes parties du corps. Pour déterminer l'émission de chaleur réelle $(Q)$ au niveau des différentes régions, il est nécessaire de faire appel à la calorimétrie particlle. Le principe de cette méthode consiste en l'isolation thermique, pendant une courte période, d'un petit volume d'air à la surface du corps. On mesure l'accroissement de température qui est fonction de la quantité de chaleur sensible émise par la peau. Il faut noter qu'on élimine les pertes de chaleur par convection puisque l'espace de mesure est fermé.

L'appareil que nous avons utilisé se compose d'une chambre de $150 \mathrm{~cm}^{2}$ de surface (P) et de I $5 \mathrm{~cm}$ de hauteur, d'un capteur de température et d'un indicateur électronique. La chambre est calorifugée au moyen d'une couche de polystyrène expansé de $4 \mathrm{~cm}$ d'épaisseur. Le capteur est constitué de 20 termocouples cuivre-constant qui donnent une indication représentative de la température moyenne de l'espace tout entier. Les soudures froides sont collées au couvercle en cluralumin de la chambre, de sorte que la température à l'intérieur est comparée, en permanence, à la température ambiante. En raison de l'inertie thermique importante du couvercle, on considère que cette température ambiante est constante pendant la durée de la mesure (quelques dizaines de secondes).

L'émission de chalcur est déterminéc au niveau de l'épaule $\left(L_{1}\right)$ de l'abdomen $\left(L_{2}\right)$ et de la glande mammaire $\left(\mathbf{L}_{\mathbf{3}}\right)$.

\section{III. - RÉSULTATS ET DISCUSSION}

\section{I. - Influence de la zone anatomique (fig. I, fig. 2)}

Les productions de chaleur, par unité de surface, sont égales au niveau de l'épaule $\left(\mathrm{L}_{1}\right)$ et de 1'abdomen $\left(\mathrm{L}_{2}\right)$. En revanche, l'émission de chaleur au niveau de la glande mammaire $\left(L_{3}\right)$ est très supérieure à $L_{1}$ et $L_{2}$, plus du double. Les différences les plus importantes sont observées avec des vaches dont la production laitière est élevée. Ce phénomène s'explique par la vascularisation importante du pis et la faible isolation thermique qu'offre sa peau presque nue. La figure 2 représente l'émission de chaleur au niveau de la glande mammaire avec les 3 races : Pie rouge, Frisonne et Ayrshire. 


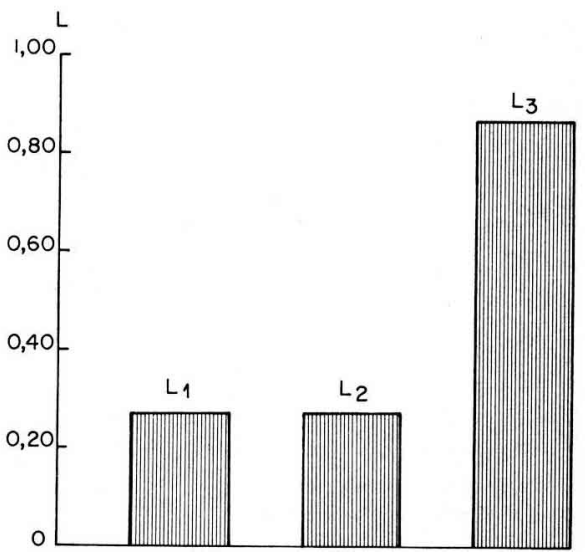

FIG. I. - Productions de chaleur en fonction de la zone anatomique

$\mathrm{L}=$ émission de la chaleur en cal $/ \mathrm{s} / \mathrm{surface}$ de $\mathrm{I} 5 \mathrm{c} \mathrm{cm}^{2}$

$\mathrm{L}_{1}=$ émission de la chaleur mesurée aux épaules

$\mathrm{L}_{2}=$ émission de la chaleur mesurée au ventre

$\mathrm{L}_{3}=$ émission de la chaleur mesurée au pis.

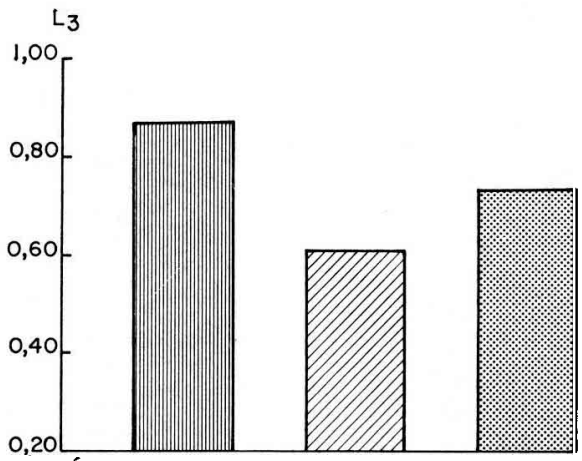

FIG. 2. - Émission de la chaleur du pis en fonction de la race

$\mathrm{L}_{3}=$ émission de la chaleur en cal $/ \mathrm{s} / \mathrm{surface}$ de $150 \mathrm{~cm}^{2}$

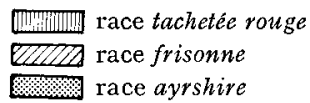

\section{2. - Infuence de la température ambiante (fig. 3)}

On a pris comme référence les productions de chaleur des vaches pour des températures ambiantes comprises entre +4 et $+16^{\circ} \mathrm{C}$ qui correspondent à la zone de neutralité thermique. Les résultats relatifs aux vaches de la race $P$ ie rouge (tabl. I) montrent que les variations individuelles sont très importantes.

Pour des températures ambiantes comprises entre -5 et $+4^{\circ} \mathrm{C}$, on n'observe pas de différence de production moyenne de chaleur au niveau de l'épaule et de l'abdomen; en revanche, l'émission de chaleur au niveau de la glande mammaire est presque doublée en passant de +4 à - 5 , ce qui montre l'action isolante du pelage.

Pour des températures ambiantes supérieures à $\mathrm{I} 6^{\circ} \mathrm{C}$, la production de chaleur par unité de surface est plus élevée pour les parties couvertes de poils que pour les parties nues. 


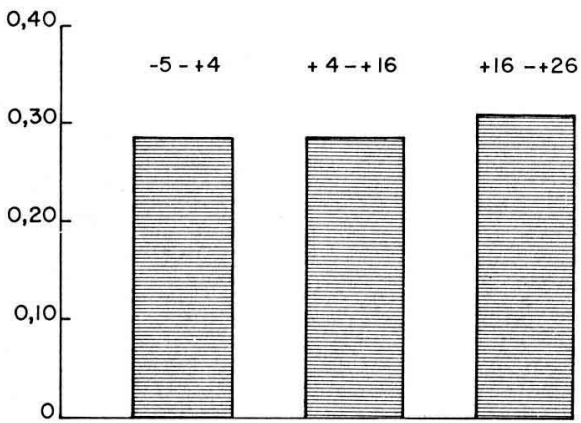

FIG. 3. - Productions de chaleur en fonction de la température ambiante $\mathrm{T}=$ température en ${ }^{\circ} \mathrm{C}$,

$L_{1}+L_{2}=$ émission de la chaleur en cal $/ \mathrm{s} / \mathrm{surface}$ de $\mathrm{I}_{5} \mathrm{o} \mathrm{cm}^{2}$.

\section{TABLEAU I}

Production de chaleur des vaches laitières de la race Pic rouge placées à des températures ambiantes comprises entre +4 et $+16^{\circ} \mathrm{C}$

\begin{tabular}{|c|c|c|c|c|}
\hline \multirow{3}{*}{$\begin{array}{c}\text { Vache } \\
\text { No }\end{array}$} & \multirow{3}{*}{$\begin{array}{l}\text { Production } \\
\text { laitière }\end{array}$} & \multicolumn{3}{|c|}{ Production de chaleur cal/s./P } \\
\hline & & \multicolumn{2}{|c|}{ Zone anatomique } & \multirow{2}{*}{$\begin{array}{c}\text { Moyenne } \\
\text { de } \mathrm{L}_{1}+\mathrm{L}_{2}\end{array}$} \\
\hline & & $\begin{array}{c}\text { Eppaule } \\
\mathrm{L}_{1}\end{array}$ & $\begin{array}{c}\text { Abdomen } \\
\mathrm{L}_{2}\end{array}$ & \\
\hline $87 !$ & & 0,335 & 0,345 & 0,340 \\
\hline 14034 & 16,8 & 0,331 & 0,422 & 0,376 \\
\hline 14092 & 9,1 & 0,313 & 0,397 & 0,355 \\
\hline 14093 & - & 0,252 & $0,214_{4}$ & 0,233 \\
\hline 14101 & 8,2 & 0,293 & 0,297 & 0,295 \\
\hline 14111 & 9,8 & 0,360 & 0,215 & 0,287 \\
\hline 14168 & 12,5 & 0,247 & 0,286 & 0,266 \\
\hline 14225 & - & 0,122 & $0,18^{\prime}$ & 0,153 \\
\hline 14256 & 8,5 & 0,216 & 0,195 & 0,206 \\
\hline \multicolumn{2}{|c|}{ Moyenne } & 0,274 & 0,284 & 0,279 \\
\hline
\end{tabular}

\section{3. - Variation de la production de chaleur des vaches laitières} en fonction de la production de lait

Les déterminations de production de chaleur ont été faites aux divers niveaux (épaule, abdomen, glande mammaire) avec des vaches placées en stabulation libre à une température ambiante de $6^{\circ} \mathrm{C}$. Les résultats regroupés dans le tableau 2 montrent que l'émission de chaleur par unité de surface au niveau de la glande mammaire augmente avec la production laitière.

Chez les vaches de race laitière (Frisonne et Ayrshire) l'émission de chaleur par unité de surface au niveau de l'épaule et de l'abdomen est supérieure à celle des races mixtes (Pie rouge) (tab1. 2 et 3 ). 
TABLEAU 2

Production de chaleur des vaches laitières de race Pie rouge à différents niveaux de production laitière

(Moyennes de $\mathrm{L}_{1}$ et $\mathrm{L}_{2}$ )

\begin{tabular}{c|c|c|c|c|c}
\hline $\begin{array}{c}\text { Vache } \\
\text { No }\end{array}$ & $\begin{array}{c}\text { Vache } \\
\text { taries* }\end{array}$ & $\begin{array}{c}\text { Vache } \\
\text { No }\end{array}$ & $\begin{array}{c}\text { Production infé- } \\
\text { rieure à 10 kg* }\end{array}$ & $\begin{array}{c}\text { Vache } \\
\text { No }\end{array}$ & $\begin{array}{c}\text { Production supé- } \\
\text { rieure à 25 kg* }\end{array}$ \\
\hline 874 & 0,330 & 6,23 & 0,293 & 14031 & 0,281 \\
14058 & 0,479 & 14011 & 0,330 & 14044 & 0,430 \\
14093 & 0,233 & 14092 & 0,221 & 14050 & 0,502 \\
14271 & 0,297 & 14111 & 0,288 & 14092 & 0,466 \\
& 22150 & 0,264 & 14112 & 0,287 \\
\hline
\end{tabular}

* Production de chaleur (cal/s/surface de $150 \mathrm{de} \mathrm{cm}^{2}$ )

\section{TABLEAU 3}

Production de chaleur des vaches laitières des races Frisonne et Ayrshire $\grave{a}$ des températures ambiantes comprises entre +4 et $16^{\circ} \mathrm{C}$

\begin{tabular}{|c|c|c|c|c|c|c|}
\hline \multirow{2}{*}{ Race } & \multirow{2}{*}{$\begin{array}{c}\text { Nombre } \\
\text { d'animaux }\end{array}$} & \multirow{2}{*}{ Poids moyen } & \multirow{2}{*}{$\begin{array}{l}\text { Production } \\
\text { laitière } \\
\text { moyenne }\end{array}$} & \multicolumn{3}{|c|}{ Production de chaleur cal/s/P } \\
\hline & & & & $\begin{array}{c}\mathrm{L}_{1} \\
\text { Épaule }\end{array}$ & $\underset{\text { Abdomen }}{\mathrm{L}_{2}}$ & $\begin{array}{c}\text { Moyenne } \\
\mathrm{L}_{1}+\mathrm{L}_{2}\end{array}$ \\
\hline Ayrshire & 10 & 490 & 12,3 & 0,343 & 0,290 & 0,317 \\
\hline Frisonne & 10 & 570 & 14,8 & 0,408 & 0,366 & 0,387 \\
\hline Mloyenne & & & & 0,372 & 0,325 & 0,349 \\
\hline
\end{tabular}

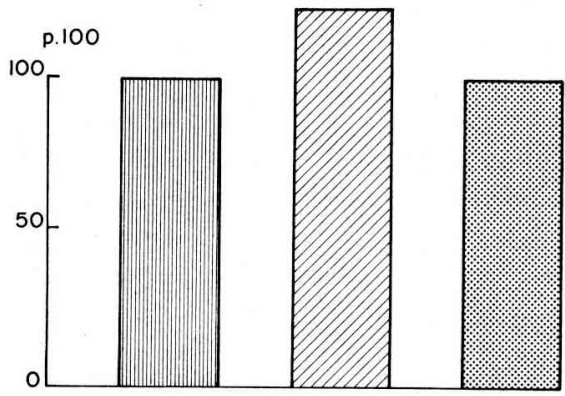

FIG. 4. - Productions de chaleur en fonction de la race 


\section{4. - Variations de la production de chaleur des vaches laitières en fonction de la race (fig. 4)}

Les productions moyennes de chaleur au niveau de l'épaule et de l'abdomen des vaches laitières placées à des températures ambiantes comprises entre +4 et $+{ }^{\circ} 6^{\circ} \mathrm{C}$ (tab1. 3) sont plus élevées chez les vaches de la race Frisonne que chez les vaches des races $P$ ie rouge et Ayrshire. Il faut noter cependant que la production laitières des vaches Frisonnes par unité de poids vif, est supérieure de 23 p. Ioo à celle des vaches des autres races.

\section{5. - Production de chaleur des vaches laitières en stabulation libre et en stabulation entravée}

L,es mesures ont été effectuées sur des vaches de race Pie rouge placées en stabulation libre à une température ambiante moyenne de $6,8^{\circ} \mathrm{C}$ et d'autres placées en stabulation entravée dans une étable traditionnelle dont la température ambiante moyenne était de $I 8,4^{\circ} \mathrm{C}$. Les résultats (tabl. 4) montrent que l'émission de chaleur

\section{TABLEAU 4}

Différences de production de chaleur de vaches laitières de race Pie rouge dans des conditions de stabulation différentes

\begin{tabular}{|c|c|c|c|}
\hline \multicolumn{2}{|c|}{ Stabulation libre } & \multicolumn{2}{|c|}{ Stabulation entravée } \\
\hline $\begin{array}{c}\text { Vache } \\
n^{\circ}\end{array}$ & $\begin{array}{c}\text { Production } \\
\text { de chaleur moyenne } \\
\left(\mathrm{L}_{1}+\mathrm{L}_{2}\right)\end{array}$ & $\begin{array}{c}\text { Vache } \\
n^{\circ}\end{array}$ & $\begin{array}{c}\text { Production } \\
\text { de chaleur moyenne } \\
\left(\mathrm{L}_{1}+\mathrm{I}_{\mathrm{r}_{2}}\right)\end{array}$ \\
\hline 14024 & 0,282 & 874 & 0,380 \\
\hline 14031 & 0,281 & 14079 & 0,293 \\
\hline $1 / 4034$ & 0,272 & 14085 & $0,1,196$ \\
\hline 14042 & 0,233 & 14089 & 0,195 \\
\hline 14044 & 0,430 & $1 / 4092$ & 0,283 \\
\hline 14050 & 0,502 & 1'⿺093 & 0,233 \\
\hline
\end{tabular}

au niveau de l'épaule et de l'abdomen est rarement plus élevée chez les vaches placées en stabulation libre que chez celles placées dans une étable traditionnelle; (chez certains individus on observe le contraire). Les résultats trouvés diffèrent un peu de la valeur généralement citée de neutralité thermique des bovins, indiquée dans $1^{\prime}$ introduction de cet article (entre +4 et $\left.+\mathrm{I} 6^{\circ} \mathrm{C}\right)$.

\section{6. - Calcul de la production totale de chaleur des vaches laitières à partir des résultats obtenus par calorimétrie partielle. Comparaison avec les données bibliographiques.}

L'émission de chaleur n'est pas uniforme sur toute la surface corporelle mais il est techniquement impossible de la mesurer aux extrémités, par exemple. Nous avons, cependant, essayé de calculer la production de chaleur des vaches laitières 
en utilisant les données bibliographiques concernant la surface corporelle et les coefficients de production thermique.

Selon BURĚ̀ (I965), les surfaces des vaches d'après leur poids sont données dans le tableau 5 .

TABLEAU 5

Surfaces corporelles selon les poids

\begin{tabular}{c|c|c|c|c}
\hline \hline Poids en $\mathrm{kg} \ldots$ & 450 & 500 & 550 & 600 \\
\hline Surface en $\mathrm{m}^{2} \ldots$ & 6,2 & 6,6 & 7 & 7,5 \\
\hline
\end{tabular}

Les coefficients de production thermique (HAUPTMAN, I963), suivant la zone corporelle de la race, sont indiqués dans le tableau 6.

TABLEAU 6

Répartition des surfaces corporelles et coefficients de production thermique correspondants

\begin{tabular}{|c|c|c|c|}
\hline & \multicolumn{2}{|c|}{ Races } & \multirow{2}{*}{$\begin{array}{l}\text { Coefficient } \\
\text { de production } \\
\text { thermique }\end{array}$} \\
\hline & $\begin{array}{c}\text { Pie ronge } \\
\%\end{array}$ & $\begin{array}{c}\text { Frisonne } \\
\text { 4yrshire } \\
\%\end{array}$ & \\
\hline Tête & 5 & 4 & 1,3 \\
\hline Tronc ..... & 80 & 80 & 1,0 \\
\hline Extrémités & 12 & 12 & 0, ; \\
\hline Mamelle ... & 3 & 4 & 3,2 \\
\hline
\end{tabular}

Selon BurEšs (I965), dans la zone de neutralité thermique, la chaleur latente représente $37 \mathrm{p}$. Ioo de la chaleur totale émise.

A partir des émissions de chaleur déterminées expérimentalement au niveau de l'épaule $\left(L_{1}\right)$ et de l'abdomen $\left(I_{12}\right)$ on peut estimer la production totale de chaleur d'une vache Pie rouge à $\mathrm{I} 54 \mathrm{kcal} /$ heure/Ioo $\mathrm{kg}$ de poids vif et celle d'une vache Frisonne ou Ayrshire à I9o kcal/Ioo $\mathrm{kg}$ de poids vif/heure, dans la zone de neutralité thermique.

\section{IV. - DISCUSSION E'T CONCLUSION}

I a méthode de calorimétrie partielle utilisée apparaît comme une méthode convenable pour l'étude poursuivie. Elle a permis en effet d'obtenir des résultats grâce à des mesures de courte durée, sans influencer défavorablement les animaux ; la précision de la méthođe choisie répond au but visé. 
Les inconvénients de la méthode sont liés à son principe : la calorimétrie partielle ne permet la détermination de l'émission de la chaleur qu'à partir de surfaces du corps déterminées, et seulement quelques-unes de ses composantes. Cependant, cet inconvénient est compensé par des avantages : il est possible d'effectuer les mesures sur une petite surface, sans stress physiologique des animatux, et d'utiliser cet appareil sur le terrain. En outre, la calorimétrie partielle permet, grâce à la précision des mesures et à la sensibiblité suffisante de l'appareillage, des mesures de courte durée ; une fois le capteur étalonné, on peut l'utiliser pour toutes les espèces d'animaux de ferme.

Les observations effectuées chez les vaches laitières placées en stabulation libre couverte montrent que sous réserve d'une bonne acclimatation des animaux il n'y a pas lieu de craindre un accroissement très sensible de l'émission de chaleur au cours de la période hivernale, ce qui entraînerait une augmentation de l'ingestion d'aliments.

Reçu pour publication en mars 1972.

\section{SUMMARY}

BIOCLIMATOLOGY IN DAIRY COWS : DESCRIPTION OF A NEW METHOD

A method of partial calorimetry for dairy cows is described, allowing short-lasting measurements at various levels, shoulder, udder, abdomen.

The mammary gland emits more heat than the other parts studied (fig. I), but this heat emission varies much according to room-temperature.

In dairy cows with a high milk yield, the heat production is greater at the level of the shoulder and abdomen than in cows with a small milk yield.

Frisian cows emit more heat than Ayrshire and Red spotted cows.

The method proposed is simple and can be used even in rudimentary installations.

\section{RÉFÉRENCES BIBLIOGRAPHIQUES}

BUREš R., I965. Přispěvek k možnosti měřeni v̀ydeje tepla s povrchu těl hospodařských zvířat. Meteorol. Zpravy, 2.

Hauptman J., 1963. Zúlklady bioklimatologie ve vztahu k ustájení. Stud. infor. U. V. T. Il. Żiv. výroba, 8, 447-527. 